\title{
Demonstration of a Discretely Tunable III-V/SOI Sampled Grating Distributed Feedback Laser
}

\author{
Sören Dhoore*, Lianyan Li, Amin Abbasi, Gunther Roelkens and Geert Morthier \\ Photonics Research Group, Department of Information Technology, Ghent University - imec, Ghent, Belgium \\ Center for Nano- and Biophotonics (NB-Photonics), Ghent University, Ghent, Belgium \\ *Email: Soren.Dhoore@UGent.be
}

\begin{abstract}
A novel III-V/SOI sampled grating DFB laser is experimentally demonstrated. Two input currents allow wavelength tuning over a $55 \mathrm{~nm}$ wide range in discrete wavelength steps of $5 \mathrm{~nm}$. A side mode suppression ratio larger than $33 \mathrm{~dB}$ is obtained for all wavelength channels.
\end{abstract}

\section{INTRODUCTION}

With the increasing demand for bandwidth, widely tunable laser diodes are expected to play an increasingly important role as light sources in future advanced data center and metro networks. Additional functionality in flexible optical network architectures can for instance be achieved through fast bandwidth relocation by dynamically increasing the number of links between hot spots in the network [1]. Optical switches or cross connects that can be used for this purpose require very fast and widely tunable laser diodes with high power efficiency [2].

Recently, significant research has been done in the area of IIIV/SOI laser diodes, in which laser structures are realised through integration of III-V epitaxy on the SOI platform through direct or adhesive wafer bonding techniques [3]. In this way the laser devices can be co-integrated with silicon passive waveguide circuits, high-speed silicon modulators and germanium photodetectors. As opposed to traditional InP-lasers, membrane III-V/SOI lasers allow the realization of gratings with a high coupling coefficient and strong optical confinement in the gain material.

Different III-V/SOI tunable lasers have been demonstrated already. These laser structures typically make use of one or more ring resonator filters with heaters that allow to thermally tune the lasing wavelength [4], [5]. An alternative laser structure that consists of 2 sampled grating DBR mirrors, a gain and a phase section, has been used to demonstrate over $13 \mathrm{~nm}$ wavelength tuning [6].

In this paper, we present the experimental demonstration of a novel widely tunable sampled grating distributed feedback (SG-DFB) laser integrated on SOI. The laser is tunable over a wavelength range larger than $55 \mathrm{~nm}$ in discrete wavelength steps of $5 \mathrm{~nm}$, with all wavelength channels having a side mode suppression ratio (SMSR) of more than $33 \mathrm{~dB}$.

\section{DESIGN \& FABRICATION}

The layout of the SG-DFB laser is schematically shown in Figure 1. The laser structure is implemented as a two-section DFB laser and constitutes a III-V gain region that is adhesively bonded on top of SOI waveguide structures with sampled gratings patterned on top. The gratings are defined in a $400 \mathrm{~nm}$ thick silicon device layer with an etch depth of $180 \mathrm{~nm}$. A different sampling period in both grating sections $(72 \mu \mathrm{m}$ and $80 \mu \mathrm{m})$ is chosen such that the Vernier effect can be exploited for wide wavelength tuning, through a variation of the injection current into the two sections. The gratings are $3.5 \mu \mathrm{m}$ wide and have a period of $480 \mathrm{~nm}$ with a duty cycle of $75 \%$. This corresponds to a Bragg wavelength of $1565 \mathrm{~nm}\left(n_{\mathrm{eff}}=3.26\right)$. The

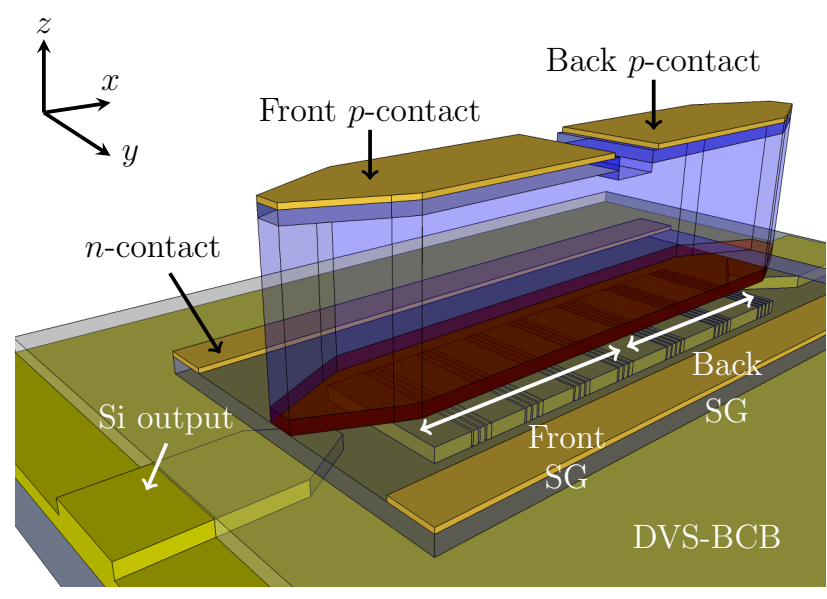

Fig. 1. Layout of the SG-DFB laser. The front and back SG have a slightly different sampling period to exploit the Vernier effect for wide wavelength tuning.

sampling duty cycle $d_{\mathrm{SG}}$ is chosen to be $10 \%$, as this ensures a sufficiently flat envelope of the overall reflection spectrum needed for a high side mode suppression across the entire tuning range [7]. The designed free spectral range (FSR) of the front grating reflector is $5 \mathrm{~nm}$, whereas the designed FSR of the back grating reflector is $4.5 \mathrm{~nm}$.

The III-V epitaxial layer stack constitutes a standard amplifier stack with an $n$-InP bottom cladding (190 nm thick), 2 InGaAsP SCH layers (100 nm thick, $1.17 \mu \mathrm{m}$ bandgap wavelength), 6 InGaAsP quantum wells ( $7 \mathrm{~nm}$ thick, $1.55 \mu \mathrm{m}$ bandgap wavelength), a $p$ InP top cladding $(1.5 \mu \mathrm{m}$ thick $)$ and a $p^{++}$-InGaAs top contact layer (200 $\mathrm{nm}$ thick). Coupling between the III-V layer and the SOI output waveguide is realised through a standard double adiabatic tapered coupler, as demonstrated in [8].

Fabrication of the SG-DFB laser is similar to the fabrication of a standard III-V/SOI DFB laser [8] and involves adhesive DVS-BCB bonding of the III-V epitaxy on the SOI waveguide structures. After III-V substrate removal the laser structure is defined through a series of lithographic, wet/dry etching and metallisation steps. The $p$-contact consists of two individual contacts that allow independent current injection in the two sampled grating sections.

A transverse and longitudinal cross-sectional SEM image of the fabricated SG-DFB laser are shown in Figures 2 (a) and (b) respectively. The laser mesa has a $\mathrm{V}$-shape, which is realised through wet etching of the top $p$-InP cladding. This enhances the optical confinement factor in the MQWs $(12 \%$ confinement factor in the 6 quantum wells) and realizes narrow taper tips. The DVS-BCB 


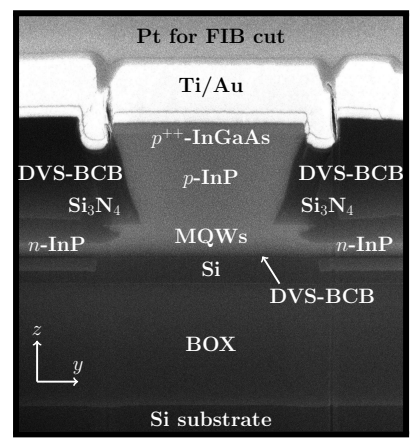

(a)

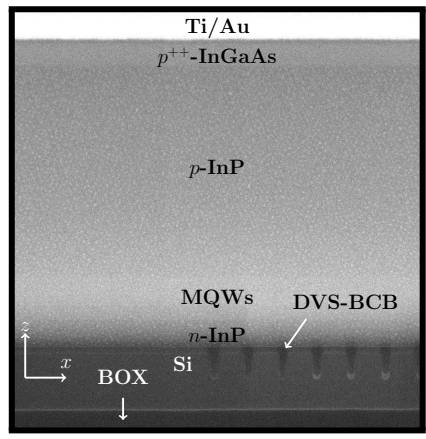

(b)

Fig. 2. SEM image of the fabricated SG-DFB laser device. (a) Transverse cross-sectional view; (b) Longitudinal cross-sectional view.

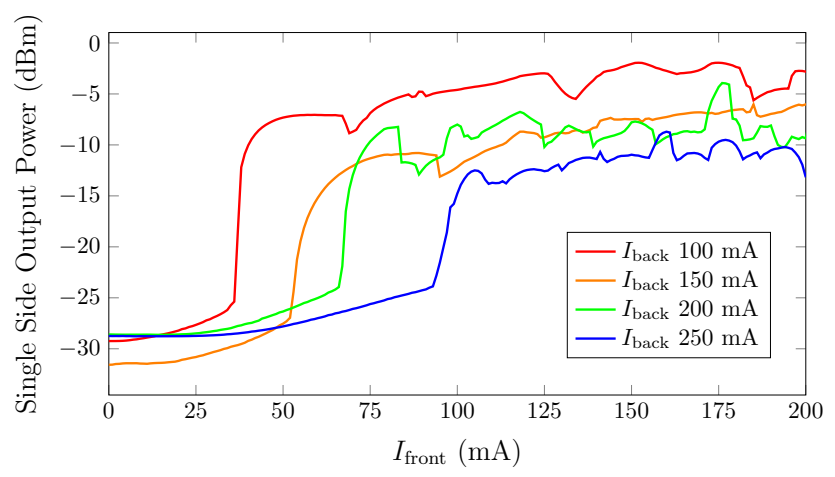

Fig. 3. Light-current $(L I)$ characteristics of the SG-DFB laser.

bonding layer thickness is only $40 \mathrm{~nm}$, which gives rise to a rather large effective grating coupling constant $\kappa_{\mathrm{eff}}=\kappa \cdot d_{\mathrm{SG}}$ of $31.8 \mathrm{~cm}^{-1}$.

\section{MEASUREMENT Results}

Characterisation of the laser is done at a stage temperature of $10{ }^{\circ} \mathrm{C}$. A standard fiber-to-chip SOI grating coupler is used to couple the laser light to a standard single-mode optical fiber. Using a current source and three electrical DC probes the front $\left(I_{\text {front }}\right)$ and back $\left(I_{\text {back }}\right)$ grating section are independently biased.

Figure 3 shows the light-current $(L I)$ characteristics of the SGDFB laser. Thereby $I_{\text {front }}$ is swept while $I_{\text {back }}$ is kept constant. For uniform pumping $\left(I_{\text {front }}=I_{\text {back }}\right)$ the total threshold current is $90 \mathrm{~mA}$. At $\left(I_{\text {front }}, I_{\text {back }}\right)=(175 \mathrm{~mA}, 100 \mathrm{~mA})$ the fiber-coupled output power is $-1.94 \mathrm{dBm}$. The ripples in the $L I$-curves are attributed to parasitic reflections from the grating couplers.

Figure 4 (a) shows the superimposed lasing spectra of the different achievable wavelength channels across the entire tuning range. Lasing occurs from 1547 to $1603 \mathrm{~nm}$, at a discrete number of wavelengths. The spacing between these wavelength channels is $5 \mathrm{~nm}$ and corresponds with the designed peak spacing. Around each wavelength channel about $1.5 \mathrm{~nm}$ quasi-continuous tuning is possible. Figure 4 (b) depicts the fiber-coupled output power and SMSR for all different wavelength channels. All operate in single-mode regime with a SMSR ranging from $33 \mathrm{~dB}$ to $48 \mathrm{~dB}$. The fiber-coupled output power varies from $-12 \mathrm{dBm}$ to $-2.8 \mathrm{dBm}$. The variation in the fibercoupled output power is due to the variation of the total current for all

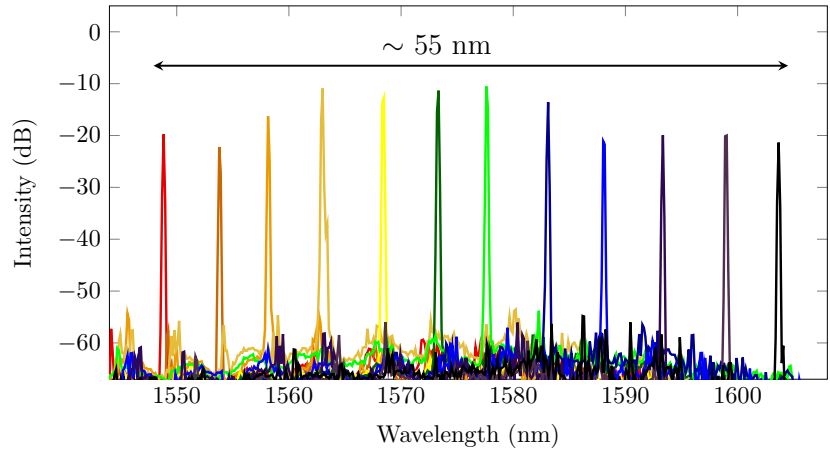

(a)

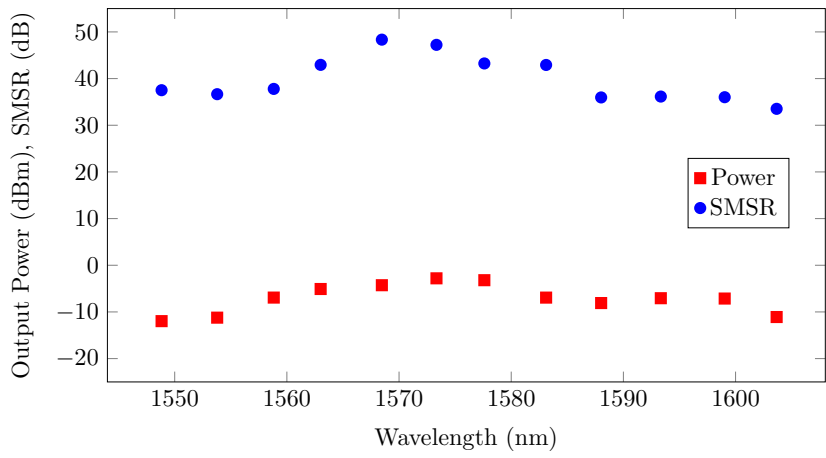

(b)

Fig. 4. Tuning characteristics. (a) Superimposed lasing spectra; (b) Fibercoupled output power and SMSR at the different wavelength channels.

wavelength channels and due to the influence of the grating coupler response.

\section{CONCLUSION}

In summary, we have presented the experimental demonstration of a novel heterogeneously integrated III-V/SOI sampled grating distributed feedback laser. With only two input currents the laser device is discretely tunable over a wide wavelength range of $55 \mathrm{~nm}$ in steps of $5 \mathrm{~nm}$. All wavelength channels operate in single-mode regime with a SMSR larger than $33 \mathrm{~dB}$.

\section{REFERENCES}

[1] Z. Cao et al., "Experimental demonstration of flexible bandwidth optical data center core network with all-to-all interconnectivity," Journal of Lightwave Technology, vol. 33, no. 8, pp. 1578-1585, 2015.

[2] C. Kachris et al., Optical interconnects for future data center networks. Springer Science \& Business Media, 2012.

[3] G. Roelkens et al., "III-V-on-silicon photonic devices for optical communication and sensing," in Photonics, vol. 2, no. 3. Multidisciplinary Digital Publishing Institute, 2015, pp. 969-1004.

[4] J. Hulme et al., "Widely tunable Vernier ring laser on hybrid silicon," Optics Express, vol. 21, no. 17, pp. 19718-19722, 2013.

[5] S. Keyvaninia et al., "Demonstration of a heterogeneously integrated IIIV/SOI single wavelength tunable laser," Optics Express, vol. 21, no. 3, pp. 3784-3792, 2013.

[6] M. N. Sysak et al., "Integration of hybrid silicon lasers and electroabsorption modulators," Optics Express, vol. 16, no. 17, pp. 12478-12486, 2008.

[7] J. Buus et al., Tunable laser diodes and related optical sources. WileyInterscience New York, 2005.

[8] S. Keyvaninia et al., "Heterogeneously integrated III-V/silicon distributed feedback lasers," Optics Letters, vol. 38, no. 24, pp. 5434-5437, 2013. 